\title{
Research on AO/FO batch management technology in aircraft production
}

\author{
Haijun Yin ${ }^{1, \mathrm{a}^{*}}$, Yuning Wang ${ }^{1, \mathrm{~b}}$, and Yingji Shan $^{1 \mathrm{c}}$ \\ 1Shenyang Aircraft Industry (Group) Co., Ltd. No.1 Lingbei St HuangGu district, Shenyang, China

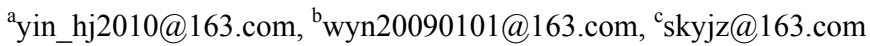

\begin{abstract}
Based on the analysis of the characteristics and significance of $\mathrm{AO} / \mathrm{FO}$ in the process of aircraft production, this paper analyzes the format rules of $\mathrm{AO} / \mathrm{FO}$ batch management from the perspective of technology realization, and details the $\mathrm{AO} / \mathrm{FO}$ The change of the query and the change status tracking, introduces the $\mathrm{AO} / \mathrm{FO}$ single-stand status display in the batch management, increases the structure definition of the attribute table in the batch management, and designs the relevant algorithm to store and calculate the batch information. Finally, based on the above theory support $\mathrm{AO} / \mathrm{FO}$ batch management system successfully used in the production of a machine.
\end{abstract}

\section{Introduction}

Influenced by the small batch and the variety of production methods, the aircraft product production organization is put into production by batch, and the assembly process is organized according to the production. The technical state of the production is concentrated in assembly, and the main basis of product assembly is the assembly outline, namely AO. $\mathrm{AO}$ is an instructional process document which is written by the technical department according to the requirements of assembly technique, process level and quality assurance[1]. MBOM is based on separation process surface based on product batch of vehicles management of manufacturing product structure view[10], some different types of aircraft sorties of MBOM may not be the same, before generated by the product code and batch of vehicles to find suitable for the vehicles of $\mathrm{AO} / \mathrm{FO}[2-3]$. It also requires effective management of all kinds of data in the aircraft's batch production[3-4].

The development of early aircraft products is mainly based on single configuration, and simultaneously is affected by the first generation of CAPP software architecture. The aircraft manufacturers mainly manage $\mathrm{AO} / \mathrm{FO}$ through the latest version of valid form. With the promotion of serialized and configured development mode of aircraft manufacturing, the traditional $\mathrm{AO} / \mathrm{FO}$ management mode is no longer suitable for the management and control of aircraft technical state. From the technical realization perspective, this paper discusses the realization mode of $\mathrm{AO} / \mathrm{FO}$ management from valid management of latest version to valid management of multiple versions based on shipset,

\footnotetext{
*Corresponding author: yin_hj2010@163.com
} 
and successfully applies this mode in the production process of a certain type of batch-produced aircraft.

\section{AO/FO batch management and its rules}

\subsection{Meaning of batch management}

In a traditional CAPP system, there are multiple versions of AOs/FOs under the same component number, but the latest version is valid. If the aircraft from different shipset are produced simultaneously in a development process, the valid shipset management is required to be performed on different versions of AOs/FOs to realize the association of revision with batch and the simultaneously-valid multiple versions, thereby effectively guiding the production and assembly of parts from different batch, to guarantee the correctness and uniqueness of production data.

When $\mathrm{AO} / \mathrm{FO}$ is required to be changed, the compiler has mastered the information about aircraft batch concerned with this change, and the corresponding valid $\mathrm{AO} / \mathrm{FO}$ requires to be solved by means of the logical operation of batch validity management module of CAPP system. Because the multiple versions of AOs/FOs may be concerned in each change, this is a complicated recording process, and therefore, it is required that a change process must be traceable, which enables users to master the change process, functioning as prevention of change missing.

$\mathrm{AO} / \mathrm{FO}$ batch management is also the management of aircraft configuration, and in the aircraft configuration, the principle that the latest revision is valid [4] is adopted, that is, when different batch of $\mathrm{AOs} / \mathrm{FOs}$ are changed, the highest version of $\mathrm{AO} / \mathrm{FO}$ of components in this shipset is the valid one. Reasonable management of $\mathrm{AO} / \mathrm{FO}$ batch validity will aid in the improvement of the ability to manage and control aircraft state, such that the problem of design and manufacturing is disposed more accurately and effectively [5-6].

\subsection{Batch management format rules}

When inputting validity, necessary batch format rules must be agreed, to realize unified data rules, which functions as calculation and query. Table 1 lists several batch management forms.

Table 1. Several AO/FO Batch Management Forms

\begin{tabular}{|c|c|c|}
\hline No. & Batch Form & Batch Format Writing Rules. \\
\hline 1 & Only & According to configured bits, formatted in figures. \\
\hline 2 & From & $\begin{array}{c}\text { According to configured bits, formatted in figures and } \\
\text { English symbol "-". }\end{array}$ \\
\hline 3 & Segment & $\begin{array}{c}\text { According to configured bits, formatted in figures and } \\
\text { English symbol "-". }\end{array}$ \\
\hline
\end{tabular}

"Only" indicates this $\mathrm{AO} / \mathrm{FO}$ is only applicable to the production or assembly of the batch components;

"From" indicates this AO/FO is applicable to the production or assembly of this batch and its subsequent components;

"Segment" indicates this AO/FO is applicable to the production or assembly of the batch components within a range. 
Batch information may be any one of the above three conditions to represent the only meaning, and may be any combination of the above three conditions to represent multiple meanings.

\section{Changing and tracking of AO/FO batch information}

\subsection{Change}

The query of AO/FO of a CAPP system component may be performed by the operator according to number localization or changing basis. According to different product processing states, the searched result may be divided into three conditions:

(1) When a product belongs to a manufactured product from its processing state, the system displays all query results of the product, the compiler identifies the corresponding shipset (version) of $\mathrm{AO} / \mathrm{FO}$, works out the rework and repair $\mathrm{AO} / \mathrm{FO}$, and assigns the batch information to it;

(2) When a product belongs to an in-process product from its processing state, the system displays all query results of the product, the compiler identifies the corresponding shipset (version) of $\mathrm{AO} / \mathrm{FO}$, creates a temporary amendment sheet, and assigns the batch information to it;

(3) When a product belongs to a product to be manufactured from its processing state, the system displays all query results of the product, the compiler identifies the corresponding shipset (version) of $\mathrm{AO} / \mathrm{FO}$, needs to update the original $\mathrm{AO} / \mathrm{FO}$, computes the recommended batch according to logical algorithm, and assigns the batch information to it[7-8].

\subsection{Tracking of change state}

The compiler can look up the change state and the change process by locating $\mathrm{AO} / \mathrm{FO}$ number and version, and gives a feedback for updating condition of a component's $\mathrm{AO} / \mathrm{FO}$ to the compiler, including unchanged batch, changed batch, time of change, and changed version.

\section{Single sortie state displaying}

By displaying the single sortie state, the directory information display that AOs/FOs of a certain model of aircraft in a shipset are all effective is realized, mainly including two aspects of content.

(1) When displaying the single sortie state, the aircraft model, compilation unit and compiler corresponding to $\mathrm{AO} / \mathrm{FO}$ are involved, and the authority of $\mathrm{AO} / \mathrm{FO}$ department is divided and positioned;

(2) The single sortie state may also be inquired and displayed by taking other process documents as input conditions, such as temporary amendment sheet, matching card, technical sheet, rework and repair $\mathrm{AO} / \mathrm{FO}$, as well as by means of $\mathrm{AO} / \mathrm{FO}$ type (general, important and critical).

\subsection{Query condition setting of single sortie state}

The set query conditions mainly include model, batch, compilation department, compiler, type of process document, basis of compilation, etc., wherein the model is automatically loaded by the system, and is not editable in general; the batch is input by the operator, with 
the format rule automatically judged by the system. the department and the compiler are usually constant, and can be selectively input by the operator; process documents may include part number, matching card, temporary amendment sheet, technical sheet, rework and repair $\mathrm{AO} / \mathrm{FO}$, etc., one or more of which can be selected by the operator. The basis of compilation may be divided into process documents, process improved contents or other documents, one or more of which can be selected by the operator[9-10].

\subsection{Query result of single sortie state}

The system performs logical operations based on the set query condition to query out $\mathrm{AO} / \mathrm{FO}$ and process documents for this batch within the range of the condition. The results of querying a directory mainly include $\mathrm{AO} / \mathrm{FO}$ number, $\mathrm{AO} / \mathrm{FO}$ version, document type and document name.

\section{Structural definition and logical algorithm of attribute table}

\subsection{Structural definition of database}

In this paper, it is recommended that oracle database be used for defining and storing batch management data, $\mathrm{AO} / \mathrm{FO}$ data be structured, and table structural definition be added in the database, in order to record the generation, change and final result display of all batch information and for translation display of $\mathrm{AO} / \mathrm{FO}$ batch management information. Refer to Table 2 for the structural definition of attribute table.

Table 2. Structural Definition of attribute table

\begin{tabular}{|c|c|c|c|c|}
\hline No. & $\begin{array}{c}\text { Field } \\
\text { Definition }\end{array}$ & $\begin{array}{c}\text { Field Value } \\
\text { Type }\end{array}$ & Field Meaning & Remarks \\
\hline 1 & ID & Integer (32) & Internal Sequence & $\begin{array}{c}\text { Auto-increment } \\
\text { Sequence }\end{array}$ \\
\hline 2 & PLANNO & String (64) & Number & Structured Data Value \\
\hline 3 & MODI & Integer (8) & $\begin{array}{c}\text { Internal Change } \\
\text { Times }\end{array}$ & $\begin{array}{c}\text { Auto-increment } \\
\text { Sequence }\end{array}$ \\
\hline 4 & JC & String (128) & $\begin{array}{c}\text { Batch Change } \\
\text { Requirement }\end{array}$ & Interface Value \\
\hline 5 & $\begin{array}{c}\text { VER_FRO } \\
\text { M }\end{array}$ & String (16) & $\begin{array}{c}\text { Unchanged } \\
\text { Version }\end{array}$ & Structured Data Value \\
\hline 6 & VER_NOW & String (16) & Changed Version & Computation Value \\
\hline 7 & VER_NO & String (16) & $\begin{array}{c}\text { Internal Version } \\
\text { Sequence }\end{array}$ & $\begin{array}{c}\text { Auto-increment } \\
\text { Sequence }\end{array}$ \\
\hline 8 & JC_FROM & String (128) & Unchanged Batch & Structured Data Value \\
\hline 9 & JC_TO & String (128) & Changed Batch & Computation Value \\
\hline
\end{tabular}

\subsection{Logical algorithm}

\subsubsection{Variable definition}

The number INPUT_PLANNO, minimum shipset INPUTJC_MIN and maximum shipset INPUTJC_MAX are the data input by the operator;

shipset change MODI will automatically increase with shipset change; the corresponding revision VER_FROM is from the structured data of the system; the variables 
ORA_MIN, ORA_MAX and VER_NOW are computed by system design logical algorithm.

Table 3. Attribute Table of Logical Algorithm Variable Definition of Batch Management

\begin{tabular}{|l|c|c|c|c|}
\hline No. & Data Source & Variable Meaning & Variable Name & Remarks \\
\hline 1. & $\begin{array}{c}\text { Input Dialog } \\
\text { Box }\end{array}$ & Procedure Number & INPUT_PLANNO & $\begin{array}{c}\text { Interface } \\
\text { Value }\end{array}$ \\
\hline 2. & $\begin{array}{c}\text { Input Dialog } \\
\text { Box }\end{array}$ & Minimum Batch & INPUTJC_MIN & $\begin{array}{c}\text { Interface } \\
\text { Value }\end{array}$ \\
\hline 3. & $\begin{array}{c}\text { Input Dialog } \\
\text { Box }\end{array}$ & Maximum Batch & INPUTJC_MAX & $\begin{array}{c}\text { Interface } \\
\text { Value }\end{array}$ \\
\hline 4. & Database & Batch Change & MODI & $\begin{array}{c}\text { Auto-increme } \\
\text { nt Sequence }\end{array}$ \\
\hline 6. & Database & $\begin{array}{c}\text { Historical shipset is } \\
\text { less than the maximum } \\
\text { value of INPUT_MIN. }\end{array}$ & ORA_MIN & $\begin{array}{c}\text { Computation } \\
\text { Value }\end{array}$ \\
\hline 7 & Database & $\begin{array}{c}\text { Historical shipset is } \\
\text { more than the } \\
\text { minimum value of } \\
\text { INPUT_MIN. }\end{array}$ & ORA_MAX & $\begin{array}{c}\text { Computation } \\
\text { Value } \\
\text { Revision }\end{array}$ \\
\hline 8 & Database & New Revision & VER_NOW & $\begin{array}{c}\text { Computation } \\
\text { Value }\end{array}$ \\
\hline
\end{tabular}

\subsubsection{Algorithm Flow}

Within the scope of the same number and batch change, if the changed batch (JC_TO) is less than the minimum batch (INPUTJC_MIN) or the unchanged batch (JC_FROM) is more than the maximum batch (INPUTJC_MAX), indicating no eligible batch record, it is unnecessary to upgrade the original $\mathrm{AO} / \overline{\mathrm{FO}}$, but it is necessary to establish new $\mathrm{AO} / \mathrm{FO}$ with the steps as shown in Algorithm 1; if the unchanged batch (JC_FROM) is less than or equal to the minimum batch (INPUTJC_MIN), it is necessary to upgrade the original $\mathrm{AO} / \mathrm{FO}$ as well as compute and store the corresponding batch information, and specific steps are shown in Algorithm 2. This section provides batch solving algorithms in two conditions:

Algorithm 1: Establishment of New AO/FO

Step1: Assign 0 to the AO/FO revision (VER_FROM) corresponding to the current batch;

Step2: Increase the new AO/FO revision (VER_NOW) by 1;

Step3: Assign the minimum shipset (INPUTJC_MIN) to the unchanged batch (JC_FROM);

Step4: Assign the maximum shipset (INPUTJC_MAX) to the changed batch (JC_TO);

Algorithm 2: Solving of Revision and Batch

Step1: Record AO/FO where the unchanged batch is as R1;

Step2: Regard new AO/FO revision (R1. VER_NOW) as the current AO/FO revision (VER_FROM);

Step3: Increase the new AO/FO revision (VER NOW) by 1;

Step4: Assign the minimum shipset (INPUTJC_MIN) to the unchanged batch (JC_FROM);

Step5: Compare the changed batch of R1 (R1.JC_TO) with the maximum shipset (INPUTJC_MAX); if the changed batch of R1 (R1.JC_TO) is less than the maximum 
shipset (INPUTJC_MAX), assign the changed batch of R1 (R1.JC_TO) to the newly recorded batch (JC_ $\overline{\mathrm{TO}}$ ), and assign the changed batch of $\mathrm{R} 1$ (R1.JC_TO) by 1 to the minimum shipset (IN̄PUTJC_MIN);

Step6:Compare the minimum shipset (INPUTJC_MIN) with the maximum shipset (INPUTJC_MAX); if the former is less than the latter, then do Step 1 to Step 5;

Step7: Compare the changed batch of R1 (R1.JC_TO) with the maximum shipset (INPUTJC_MAX); if the former is more than the latter, then do Step 8 to Step 12;

Step8: Assign the maximum shipset (INPUTJC_MAX) to the changed batch (JC_TO);

Step9: Assign the AO/FO revision of R1 (R1.VER_FROM) to the current AO/FO revision (VER_FROM);

Step10: Assign the new AO/FO revision of R1 (R1.VER_NOW) to the new AO/FO revision (VER_NOW);

Step11: Assign the maximum shipset (INPUTJC_MAX) increased by 1 to the unchanged batch (JC_FROM);

Step12: Assign the changed batch of R1 (R1.JC_TO) to the changed batch (JC_TO).

\section{Necessary constraints on batch change}

When the batch is changed, it is required to add the following necessary constraints on batch change to ensure change logic accuracy and data integrity:

(1) Before completion of some change, it is unable to perform next change;

(2) During some change, the leading strategy is computed by the above mentioned logical algorithm, and the change process to be performed is recorded by a method of making change identification.

\section{Conclusions}

In this paper, through the use of the above rules and algorithms, the software functions were improved, and successfully applied to the manufacture of a certain type of aircraft, to achieve the sorting and version of the match. For the same AO/FO, the latest version of $\mathrm{AO} / \mathrm{FO}$ is a valid version; for different versions of $\mathrm{AO} / \mathrm{FO}$, it may correspond to different batches, which can exist as both valid AO/FO. The implementation of this AO/FO batch planning to achieve the same batch of production sites, different parts of the production line at the same time production, and can be part of the production status of the effective tracking. This batch management method has been in a batch of aircraft production process to achieve the engineering application, improve the management of aircraft technology status, which has great technical promotion value.

\section{References}

1. Yan Yijun, Ye Wenhua, and Yang Yutu. Research and Development of Aircraft Batch Management System Based on AO [J]. China Manufacturing Informatization, 2000, 21(6): 703-706.

2. Shan Linqing. Research and Realization of Aircraft Manufacturing BOM Management Method [D]. Xi'an: Northwestern Polytechnical University. 2005.

3. Yan Yijun. Research and Development of Aircraft Assembly Batch Management System Based on AO [D]. Nanjing: Nanjing University of Aeronautics and Astronautics. 2007.

4. Li Rong, SunYan, and Jiang Haokang. Product Data Batch Validity Management [J]. Business Research, 2014, 16: 53-54. 
5. Wang Shuang. Technical Research of Aircraft Manufacturing Process Information System [D]. Xi'an: Northwestern Polytechnical University. 2006.

6. Jia Xiaoliang. Development of Process Information Integration Platform for Manufacturing Enterprises and Research of its Key Technologies [D]. Xi'an: Northwestern Polytechnical University. 2004.

7. Guo W. A Method of Dispatch Reliability Distribution and Prediction for Civil Aircraft in Development Phase[J]. Civil Aircarft Design \& Research, 2012.

8. Dongqi W U, Zhu W, Wang C, et al. Research on the Three-dimensional AO Technology for Airplane[J]. Modern Machinery, 2010.

9. Wu M C, Hsu Y K. Design of BOM configuration for reducing spare parts logistic costs[J]. Expert Systems with Applications, 2008, 34(4):2417-2423.

10. Li Y, Zhao L. Heuristic Assembly Order Design of Aircraft Based on Semantics Association[J]. Lecture Notes in Electrical Engineering, 2013, 227:263-268. 